\title{
Balance between Four Types of Synaptic Input for the Integrate-and-Fire Model
}

\author{
Jianfeng Feng* ${ }^{*} \dagger$, Guibin Li†, David Brown $\dagger$ and Hilary Buxton* \\ *COGS, Sussex University, Brighton BN1 9QH, U.K. and †Computational Neuroscience Laboratory, \\ The Babraham Institute, Cambridge CB2 4AT, U.K.
}

Received 9 November 1999; Accepted in revised form 10 October 2000

\begin{abstract}
We consider the integrate-and-fire model with AMPA, NMDA, GABA $A$ and $\mathrm{GABA}_{B}$ synaptic inputs, with model parameters based upon experimental data. An analytical approach is presented to determine when a post-synaptic balance between excitation and inhibition can be achieved. Secondly, we compare the model behaviour subject to these four types of input, with its behaviour subject to conventional point process inputs. We conclude that point processes are not a good approximation, even away from exact presynaptic balance. Thirdly, numerical simulations are presented which demonstrate that we can treat NMDA and GABA ${ }_{B}$ as DC currents. Finally, we conclude that a balanced input is plausible neither pre-synaptically nor post-synaptically for the model and parameters we employed.
\end{abstract}

(C) 2001 Academic Press

\section{Introduction}

Irregular firing patterns are observed in most central neurons in vivo and are thought of as essential to understanding the coding problem, but their origins are controversial. The integrateand-fire model, the simplest neuronal model, serves as a good example to explore the causes, as many publications have demonstrated (Feng, 1997; Feng \& Brown, 1998a, b, 2000; Feng, Brown, \& Li, 2000; Softky \& Koch, 1993; Shadlen \& Newsome, 1994). In the integrate-andfire model, synaptic inputs are usually modelled as simple point processes (i.e. instantaneous perturbations of membrane potential) with or without reversal potentials. We have considered the model with more complex inputs including renewal processes (Feng, 1997; Feng \& Brown,

\$ Author to whom correspondence should be addressed. E-mail: jf218@cam.ac.uk 1998a, b) correlated (Feng \& Brown, 2000) and non-instantaneous inputs (Feng et al., 2000). Aiming to make a direct comparison with experimental results and thus shed more light on the coding problem, in this paper, we consider the model with more biologically realistic input currents of AMPA, NMDA, GABA $A_{A}$ and $\mathrm{GABA}_{B}$ form.

We address the following issues: using parameters of these four types of synaptic input derived from the experimental data, when and how excitatory and inhibitory synaptic inputs reach an exact balance; under exactly balanced inputs, how does the integrate-and-fire model behave. The first question is trivial for the classic integrate-and-fire model where the inputs take a linear form. However, the situation becomes complex when we consider the model with AMPA, NMDA, GABA $A_{A}$ and $\mathrm{GABA}_{B}$ synaptic inputs due to their nonlinearity. In fact, it is 
necessary to distinguish balanced inputs at the pre- and post-synaptic site. An exact pre-synaptic balance does not imply an exact post-synaptic balance, and vice versa. The proposition that a neuron receives exactly balanced inputs is important for functional reasons: a system at exact balance is most sensitive to its inputs, and so easy to pick up input signals. There have been many studies on this topic (see, e.g. Brown \& Feng, 1999; Brown et al., 1999; Feng \& Brown, 1998b, 1999; Konig et al., 1996; Softky \& Koch, 1993; Shadlen \& Newsome, 1994). It is important therefore to consider the difference between preand post-synaptic exact balance. This distinction, to the best of our knowledge, has not been much studied theoretically. Pre-synaptic balance usually means that excitatory and inhibitory input rates are equal. This also means that the brain is wired in a way that a neuron easily detects changes in its input signals. Balance at post-synaptic sites most probably indicates that synapses are actively engaged in processing the information: usually transforming unbalanced pre-synaptic signals into balanced post-synaptic signals. Mathematical models might be useful tools to help determine whether post-synaptic balance is plausible.

In this paper, we first present an analytical approach to estimate when different synaptic inputs reach exact balance at the post-synaptic site. AMPA and $\mathrm{GABA}_{A}$ synaptic inputs are usually stronger than NMDA and $\mathrm{GABA}_{B}$ inputs and therefore play a dominant role for balanced EPSP and IPSP inputs. We find that with balanced pre-synaptic inputs, the resulting postsynaptic $\mathrm{GABA}_{A}$ current is weaker than AMPA current. This suggests that in order to reach an exact post-synaptic balance, we require either the total firing rate of pre-synaptic GABA neurons to be higher than that of the glutamate neurons, or that each IPSP hyperpolarizes the membrane potential more strongly than each depolarization resulting from each EPSP. We also find that, due to their intrinsic properties, $\mathrm{GABA}_{B}$ currents are easily saturated. We then consider the impact of different synaptic inputs on the behaviour of the integrate-and-fire model. Since each type of input is a non-instantaneous input, we find, as one might expect, that the mean interspike interval is longer than that of corresponding point process (instantaneous perturbation) input with reversal potentials. However, the coefficient of variation (CV) of interspike intervals exhibits a "push-pull" effect: the larger the mean firing rate, the larger is the CV. Numerical simulations show that the integrate-and-fire model, with physiologically plausible parameters, fires too slowly even before pre-synaptic exact balance is attained. We conclude that balance is plausible neither at presynaptic nor at post-synaptic sites, for the model and parameters we employed.

\section{The Models}

We first state models of the four types of synaptic input-AMPA, NMDA, GABA $A$ and $\mathrm{GABA}_{B}$-in detail (Destexhe et al., 1998). The method developed here can also be generalized to other types of synapse, such as dopamine, noradrenergic and cholinergic synapses. In (Destexhe et al., 1998, p. 23), the authors claimed that when pre-synaptic neurotransmitters are released in pulse form, analytical formulas for gating variables are available. We show here that for any given form of pre-synaptic neurotransmitter release, an analytical formula can be obtained.

In the central nervous system of vertebrates the major excitatory neurotransmitters are glutamate and asparate, and the major inhibitory neurotransmitters are $\gamma$-amino-butyric acid (GABA) and glycine (Zigmond et al., 1999). Here we concentrate on glutamate $\alpha$-amino-3-hydroxy-5-methyl-4-isoxalone propionic acid (AMPA), $N$-methyl-D-aspartic acid (NMDA) and GABAergic $\left(\mathrm{GABA}_{A}\right.$ and $\left.\mathrm{GABA}_{B}\right)$ synaptic inputs. NMDA and $\mathrm{GABA}_{B}$ are usually at least a factor of ten times slower than AMPA and $\mathrm{GABA}_{A}$ due to intrinsic kinetics of their receptors (see below).

\subsection{AMPA SYNAPSES}

We suppose that the pre-synaptic neurotransmitters arrive according to a Poisson process $x(t)$ with rate $\lambda_{A}$ with a specific form $f_{A}$, i.e.

$$
x_{A}(t)=\sum_{k=1}^{\infty} f_{A}\left(t-T_{k}\right) I_{\left\{t \geqslant T_{k}\right\}},
$$

where $T_{k}=\sum_{i=1}^{k} t_{i}$ for i.i.d. random sequences $t_{i}, i=1,2, \ldots$, and $P\left(t_{i} \leqslant t\right)=1-\exp \left(-\lambda_{A} t\right)$. 
In the sequence, we take the convention that subscripts are omitted, if it is clear from context which kind of synapse we refer to. The current received at the post-synaptic neuron is given by

$$
I_{A}=\bar{g}_{A} r\left(V-E_{A}\right)
$$

with the gating variable $r$ defined by

$$
\frac{\mathrm{d} r}{\mathrm{~d} t}=\alpha x(t)(1-r)-\beta r .
$$

Equation (1) describes the following diagram:

$$
C+T \rightleftharpoons O
$$

with forward and backward rates $\alpha$ and $\beta$, respectively, where $C(O)$ represent the close (open) state of glutamate receptors, and the concentration of $T$ is $x_{A}(t), r(1-r)$ is the fraction of the receptors in the open (close) state. Hence, eqn (1) can be analytically solved (see Fig. 1)

$$
\begin{aligned}
r(t)= & {\left[\int_{0}^{t} \alpha x(u) \exp \left(\int_{0}^{u}(\alpha x(s)+\beta) \mathrm{d} s\right) \mathrm{d} u\right] } \\
& \times \exp \left(-\int_{0}^{t}(\alpha x(s)+\beta) \mathrm{d} s\right)
\end{aligned}
$$

For simplicity of notation, we assume that the overlap between arriving neurotransmitter pulses can be neglected and the post-synaptic response is a linear summation of each response to the neurotransmitter release. It is of course only approximately true [see eqn (2) and Example 2 below]. We then can rewrite eqn (2) in the following form:

$$
r(t)=\bar{r} \sum_{k=1}^{\infty} \tilde{r}\left(t-T_{k}\right) I_{\left\{t \geqslant T_{k}\right\}}
$$
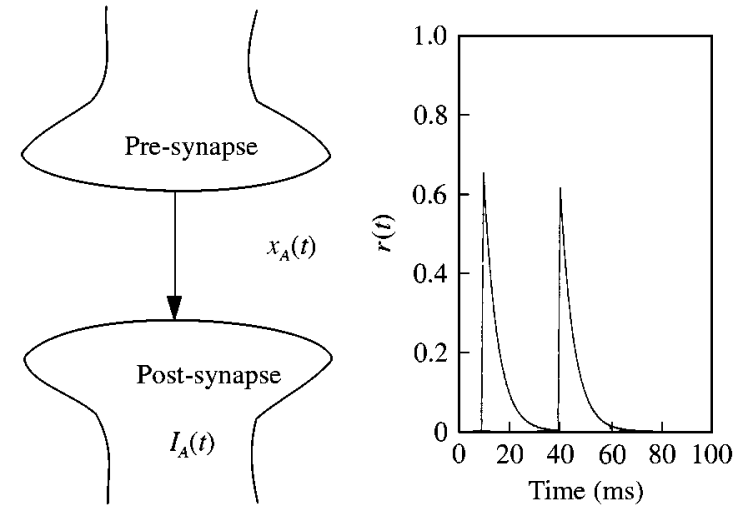

FIG. 1. Left, a schematic representation of pre-synaptic neurotransmitters $\left(x_{A}(t)\right)$ and post-synaptic currents $(r(t))$. Right, post-synaptic EPSC $r(t)$ (eqn 2) when pre-synaptic neurotransmitter arrives at time $T_{1}=10$ and $T_{2}=40 \mathrm{~ms}$ with $f_{A}(t)=\delta(t)$ (see Examples 1 and 2) and $\alpha_{A}=$ $1.1 \mathrm{~ms}^{-1} \mathrm{mM}^{-1}, \beta_{A}=0.19 \mathrm{~ms}^{-1}$.

where the basic current form $\tilde{r}(t)$ is

$$
\begin{aligned}
\tilde{r}(t)= & {\left[\int_{0}^{t} \alpha f(u) \exp \left(\int_{0}^{u}(\alpha f(s)+\beta) \mathrm{d} s\right) \mathrm{d} u\right] } \\
& \times \exp \left(-\int_{0}^{t}(\alpha f(s)+\beta) \mathrm{d} s\right) / \bar{r}
\end{aligned}
$$

with $\bar{r}=\int_{0}^{\infty} r(s) \mathrm{d} s$ as a result of the arrival of a single EPSC, i.e. it is the total charge of an arriving EPSC. Note that the basic current form $\tilde{r}(t)$ normalized, i.e. $\int \tilde{r}(t) \mathrm{d} t=1$. In the following examples, we consider two most common cases of neurontransmitter releases: release in a square wave form and an exponential form.

Example 1. When the pre-synaptic neurotransmitter is presented in square-wave (pulse) form, i.e.

$$
f\left(t-T_{k}^{E}\right)=\frac{1}{\delta} I_{\left\{T_{k}^{E}<t<T_{K}^{E}+\delta\right\}}
$$

for $\delta>0$ we have

$$
\tilde{r}(t)= \begin{cases}\frac{\alpha}{\bar{r}(\alpha+\beta \delta)}\left[\exp \left(\left(\frac{\alpha}{\delta}+\beta\right) t\right)-1\right] \exp \left(-\left(\frac{\alpha}{\delta}+\beta\right) t\right) & \text { when } t<\delta \\ \frac{\alpha}{\bar{r}(\alpha+\beta \delta)}[\exp (\alpha+\beta \delta)-1] \exp (-\alpha-\beta t) & \text { when } t \geqslant \delta\end{cases}
$$


with

$$
\begin{aligned}
\bar{r}= & \frac{\alpha}{\beta(\alpha+\beta \delta)}(1-\exp (-\alpha-\beta \delta)) \\
& +\frac{\alpha \delta}{(\alpha+\beta \delta)^{2}}[\alpha+\beta \delta-1+\exp (-\alpha-\beta \delta)]
\end{aligned}
$$

When $\delta \rightarrow 0$, i.e. $f_{A}(t)=\delta(t)$, a situation corresponding to Poisson process release of neurotransmitters, we see that

$$
\tilde{r}(t)=\frac{1}{\bar{r}}(1-\exp (-\alpha)) \exp (-\beta t)=\beta \exp (-\beta t)
$$

In this case, the neuron receives an EPSP with an instantaneous increase followed by a decay with a rate equal to $\exp (-\beta t)$ (see Fig. 1). In theoretical work, people sometimes assume that synaptic inputs simply take the form of a single exponential function. Equation (6) tells us that this is the case if we assume that pre-synaptic transmitters are very rapidly released.

Example 2. When the pre-synaptic neurotransmitter is presented in an exponential form, i.e.

$$
f(t)=a \exp (-a t)
$$

for $a>0$ we have

$$
\tilde{r}(t)=\frac{1}{\bar{r}} \alpha \exp \left(\alpha \mathrm{e}^{-a t}-\beta t\right) \int_{\mathrm{e}^{-a t}}^{1} v^{-\beta / a} \exp (-\alpha v) \mathrm{d} v
$$

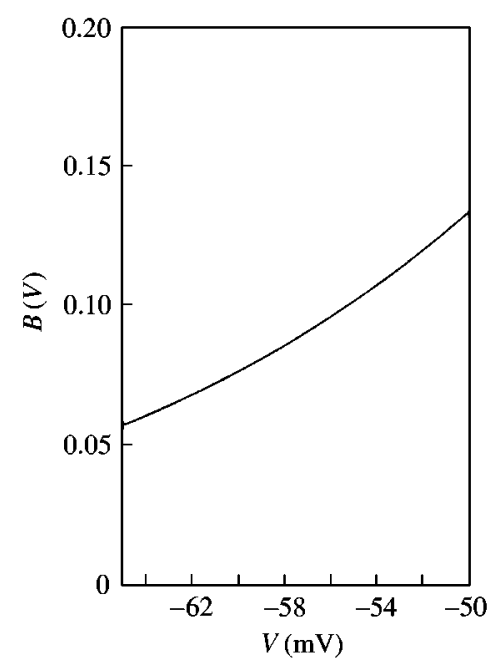

Again, when $a \rightarrow \infty$ we have

$$
\tilde{r}(t)=\frac{1}{\bar{r}}(1-\exp (-\alpha)) \exp (-\beta t)=\frac{1}{\beta} \exp (-\beta t)
$$

as we could expect (see Fig. 1). Hence, for AMPA synaptic inputs, $\alpha$ determines its magnitude, and $\beta$ is its decay rate.

\subsection{NMDA SYNAPSES}

NMDA synapses can be described by

where

$$
I_{N}=\bar{g}_{N} r B(V)\left(V-E_{N}\right)
$$

$$
B(V)=[1+\exp (-0.062 V) / 3.57]^{-1}
$$

represents the magnesium block. The gating variable $r$ is defined similar to that of AMPA synapses, with different parameters (see Appendix B). Different from AMPA synapses, $I_{N}$ nonlinearly depends on the membrane voltage of post-synaptic neurons (see Fig. 2).

\section{3. $\mathrm{GABA}_{A}$ SYNAPSES}

GABA $_{A}$ synapse can be modelled exactly as the AMPA synapse, with parameters as given in Appendix B.

\section{4. $\mathrm{GABA}_{B}$ SYNAPSES}

The most complex synapse is the $\mathrm{GABA}_{\boldsymbol{B}}$ synapse which is mediated by intracellular

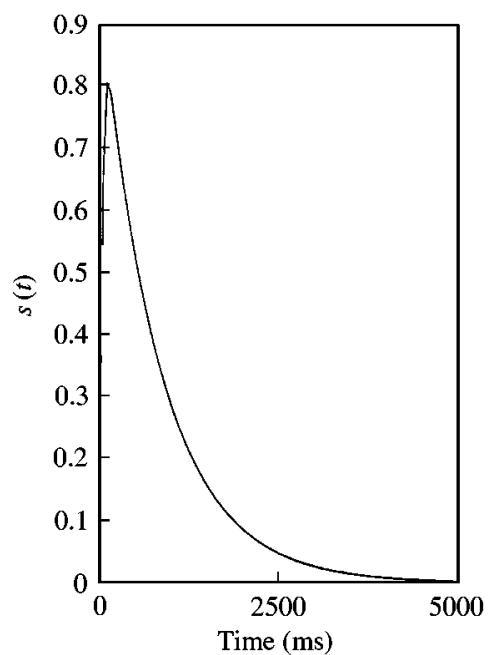

FIG. 2. Left: the nonlinear dependence of NMDA synapses on $V$. Right. $s(t)$ of $\mathrm{GABA}_{B}$ synapses when pre-synaptic neurotransmitter arrives at time $T_{1}=0 \mathrm{~ms}$ with $f_{G_{B}}(t)=\delta(t)$ (see Examples 3 and 4 and all parameters are as in Appendix B). 
second messengers (G-proteins) (Zigmond et al., 1999). $\mathrm{GABA}_{B}$ synaptic inputs result from the binding of G-proteins to open the $K^{+}$channels. As before we suppose that the pre-synaptic neurotransmitters arrive as a Poisson process $x(t)$ with rate $\lambda_{G_{B}}$ with a specific form $f$, i.e.

$$
x(t)=\sum_{k=1}^{\infty} f\left(t-T_{k}\right) I_{\left\{t \geqslant T_{k}\right\}},
$$

where $T_{k}=\sum_{i=1}^{k} t_{i}$ for i.i.d. random sequences $t_{i}$, $i=1,2, \ldots$. Then the current received at the post-synaptic neuron is given by

$$
I_{G_{B}}=\bar{g}_{G_{B}} \frac{s^{n}}{s^{n}+K_{1}}\left(V-E_{G_{B}}\right)
$$

with the gating variable $s$ defined by

$$
\frac{\mathrm{d} s}{\mathrm{~d} t}=K_{2} r-K_{3} s
$$

The basic current form $\tilde{s}(t)$ is

$$
\begin{aligned}
\tilde{s}(t)= & \frac{1}{\bar{s}} \int_{0}^{t} K_{2} \exp \left(-K_{3}(t-v)\right) \\
& \times\left\{\left[\int_{0}^{v} \alpha f(u) \exp \left(\int_{0}^{u}(\alpha f(w)+\beta) \mathrm{d} w\right) \mathrm{d} u\right]\right. \\
& \left.\times \exp \left(-\int_{0}^{v}(\alpha f(w)+\beta) \mathrm{d} w\right)\right\} \mathrm{d} v
\end{aligned}
$$

Equation (11) seems quite complex, in the following two examples we present more transparent pictures.

Example 3 (Continuation of Example 1, i.e. presynaptic transmitters are released in a pulse form). When $K_{3} \neq \beta$ we have

$$
\bar{s} \tilde{s}(t)=\frac{\alpha K_{2}}{\alpha+\beta \delta} \exp \left(-K_{3} t\right) \mid \begin{aligned}
& \frac{\exp \left(-K_{3} \delta\right)-1}{K_{3}}+(\exp (\beta \delta)-\exp (-\alpha)) \frac{\exp \left(\left(K_{3}-\beta\right) t\right)-\exp \left(\left(K_{3}-\beta\right) \delta\right)}{K_{3}-\beta} \\
& -\frac{\exp \left(-\left(K_{3}-\beta-\alpha / \delta\right) \delta\right)-1}{K_{3}-\beta-\alpha / \delta} \\
& \frac{\exp \left(-K_{3} \delta\right)-1}{K_{3}}+(\exp (\beta \delta)-\exp (-\alpha)) \\
& \times \frac{\exp \left(\left(K_{3}-\beta\right) t\right)-\exp \left(\left(K_{3}-\beta\right) \delta\right)}{K_{3}-\beta}-\delta \quad \text { when } K_{3}=\beta+\alpha / \delta,
\end{aligned}
$$

where $K_{1}, K_{2}$ and $K_{3}$ are constants, $r$ is defined as in Section 2.1, and $n$ is the independent binding sites of the $K^{+}$channels. Equation (9) can be analytically solved:

$$
\begin{aligned}
& s(t)=\int_{0}^{t} K_{2} \exp \left(-K_{3}(t-v)\right) r(v) \mathrm{d} v \\
& =\bar{r} \int_{0}^{t} K_{2} \exp \left(-K_{3}(t-v) \sum \bar{r}\left(v-T_{K}\right) I_{\left\{v \geqslant T_{k}\right\}} \mathrm{d} v\right. \\
& =\sum\left[\bar{r} \int_{T_{k}}^{t} K_{2} \exp \left(-K_{3}(t-v) \bar{r}\left(v-T_{k}\right) \mathrm{d} v\right] I_{\left\{t \geqslant T_{k}\right\}}\right. \\
& =\bar{s} \sum \tilde{s}\left(t-T_{k}\right) I_{\left\{t \geqslant T_{k}\right\}} .
\end{aligned}
$$

When $K_{3}=\beta$ we have

$$
\begin{aligned}
\bar{s} \tilde{S}(t)= & \frac{\alpha K_{2}}{\alpha+\beta \delta} \exp \left(-K_{3} t\right) \frac{\exp \left(-K_{3} \delta\right)-1}{K_{3}} \\
& +(\exp (\beta \delta)-\exp (-\alpha))(t-\delta) \\
& -\frac{\exp \left(-\left(K_{3}-\beta-\alpha / \delta\right) \delta\right)-1}{K_{3}-\beta-\alpha / \delta}
\end{aligned}
$$

In particular, when $\delta \rightarrow 0$ we see that

$$
\begin{aligned}
& \bar{s} \tilde{s}(t)=(1-\exp (-\alpha)) \\
& \times \begin{cases}\frac{K_{2}}{\beta-K_{3}}\left[\exp \left(-K_{3} t\right)-\exp (-\beta t)\right] & \text { if } \beta \neq K_{3}, \\
t \exp \left(-K_{3} t\right) & \text { if } \beta=K_{3} .\end{cases}
\end{aligned}
$$


A simple calculation shows that $\bar{s}=K_{2} /[(1-$ $\left.\exp (-\alpha))\left(K_{3} \beta\right)\right] . \bar{s} \tilde{s}(t)$ starts from zero, increases to its maximum value at $t=\left(\log K_{3}-\log \beta\right) /$ $\left(K_{3}-\beta\right)$ and then decays to zero with a rate $\exp (-\beta t)$ if $\beta<K_{3}$ (see Fig. 2).

Example 4 (Continuation of Example 2).

$$
\begin{aligned}
\bar{s} \tilde{s}(t)= & K_{2} \frac{\alpha}{a} \exp \left(-K_{3} t\right) \int_{\mathrm{e}^{-a t}}^{1} v^{\left(\left(\beta-K_{3}\right) / a\right)-1} \\
& \times \exp (\alpha v)\left(\int_{v}^{1} u^{-\beta / a} \exp (-\alpha u) \mathrm{d} u\right) \mathrm{d} v .
\end{aligned}
$$

When $\alpha \rightarrow \infty$ we see that

$$
\begin{aligned}
& \bar{s} \tilde{s}(t)=(1-\exp (-\alpha)) \\
& \times \begin{cases}\frac{K_{2}}{\beta-K_{3}}\left[\exp \left(-K_{3} t\right)-\exp (-\beta t)\right] & \text { if } \beta \neq K_{3}, \\
t \exp \left(-K_{3} t\right) & \text { if } \beta=K_{3} .\end{cases}
\end{aligned}
$$

\subsection{IF MODELS}

Suppose that when the membrane potential $V_{t}$ is between the resting potential $V_{\text {rest }}$ and the threshold $V_{\text {thre }}$, it is given by

$$
\frac{\mathrm{d} V_{t}}{\mathrm{~d} t}=-\frac{1}{\gamma}\left(V_{t}-V_{\text {rest }}\right)-I_{\text {syn }}(t)
$$

where $1 / \gamma$ is the decay rate. Synaptic input $I_{s y n}(t)$ is modelled by

$$
I_{s y n}(t)=I_{A}+I_{N}+I_{G_{A}}+I_{G_{B}} .
$$

Once $V_{t}$ pass the threshold $V_{\text {thre }}$ from below, $V_{t}$ is reset to its resting potential $V_{\text {rest }}$.

The IF model is one of the most simple neuron model which has been extensively used for modelling neuronal behaviour. Despite its simplicity, we have learnt a lot from studying it (see, e.g. Abbott et al., 1997).

\section{Results}

As mentioned in Section 1, we have to consider exactly balanced input either pre-synaptically or post-synaptically. Since we have assumed that neurotransmitter release takes the form $x(t)$ which can be easily estimated using Lemma A.1 in Appendix A, and in particular, if we assume that $\delta \rightarrow 0$ or $\alpha \rightarrow \infty$ as in Examples 1-4, then to determine when an exact pre-synaptic balance is achieved is trivial. Hence, in this paper, we restrict ourselves to exact post-synaptic balance, which is equivalent to the requirement that

$$
\left\langle I_{\text {syn }}(t)\right\rangle=0 .
$$

\subsection{AMPA AND GABA $A$ SYNAPSES}

Equation (18) reveals the difficulty when attempting to determine conditions under which exact balance can occur: the inputs to the neuron are functions of membrane potential, which means that balance cannot be achieved except at a single-membrane potential. So we rewrite the input as

$$
\begin{aligned}
\frac{\mathrm{d} V_{t}}{\mathrm{~d} t}= & -\frac{1}{\gamma}\left(V_{t}-V_{\text {rest }}\right)-I_{A}(t)-I_{G_{A}}(t) \\
= & -\left[\frac{1}{\gamma}+\bar{g}_{A} r_{A}(t)+\bar{g}_{G_{A}} r_{G_{A}}(t)\right]\left(V_{t}-V_{\text {rest }}\right) \\
& +\bar{g}_{A} r_{A}(t)\left(E_{A}-V_{\text {rest }}\right)-\bar{g}_{G_{A}} r_{G_{A}}(t)\left(V_{\text {rest }}-E_{G_{A}}\right) .
\end{aligned}
$$

Note that the first term on the right-hand side of the equation can be interpreted as a generalized decay term, the second term corresponding to input. By this means, we can define as exact post-synaptic balance at resting potential as occurring when

$$
\bar{g}_{A}\left\langle r_{A}(t)\right\rangle\left(E_{A}-V_{\text {rest }}\right)=\bar{g}_{G_{A}}\left\langle r_{G_{A}}(t)\right\rangle\left(V_{\text {rest }}-V_{G_{A}}\right) .
$$

When eqn (20) is true, a local balance between AMPA and $\mathrm{GABA}_{A}$ is attained, i.e. at the resting potential, the excitatory input current exactly balances the inhibitory input current. Since membrane potential spends much of its time in the region of the resting potential, eqn (20) might give a workable approximation to post-synaptically balanced input. 
An alternative to seeking exact balance at a specific value of membrane potential is to seek balance in a statistical sense for particular input stochastic processes. This is now discussed.

Using Lemma A.1 in Appendix A, the behaviour of $\tilde{r}(t)$ can be characterized by two phases: one is a transitional phase where $\mu(t)<1$ and the other is the stationary region where $\mu(t) \sim 1$. From Lemma 1, we conclude that a local postsynaptic exact balance at $V=V_{\text {rest }}$ is reached if and only if

$$
\lambda_{G_{A}}=\frac{\bar{g}_{A}}{\bar{g}_{G_{A}}} \frac{\bar{r}_{A}\left(E_{A}-V_{\text {rest }}\right)}{{\overline{G_{A}}}_{G_{A}}\left(V_{\text {rest }}-E_{G_{A}}\right)} \frac{\mu_{A}(t)}{\mu_{G_{A}}(t)} \lambda_{A} .
$$

Equation (21) is a very restrictive requirement which ensures that AMPA synapses are exactly balanced with $\mathrm{GABA}_{A}$ synapses, even during the transitional phase.

After the transitional phase, eqn (21) is reduced to the much simpler requirement which is easier to achieve

$$
\lambda_{G_{A}}=\frac{\bar{g}_{A}\left(V_{\text {rest }}-E_{A}\right)}{\bar{g}_{G_{A}}\left(V_{\text {rest }}-E_{G_{A}}\right)} \frac{\bar{r}_{A}}{\bar{r}_{G_{A}}} \lambda_{A}
$$

In general (see below)

$$
V_{\text {rest }}-E_{G_{A}}<E_{A}-V_{\text {rest }} .
$$

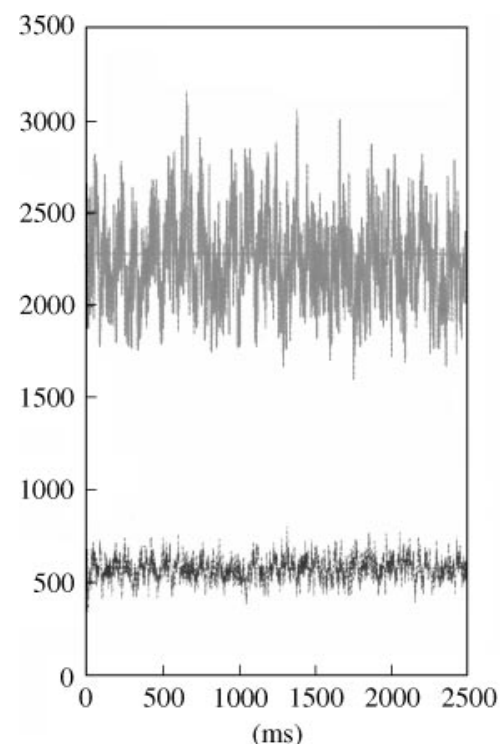

Therefore, if we assume that $g_{G_{A}}=\bar{g}_{A}$, exact balance requires that $\lambda_{G_{A}}>\lambda_{A}$ (see below) possibly much bigger because of the great discrepancy in reversal potentials. Taking account of the anatomical data that the ratio between the number of inhibitory synapses and excitatory synapses is $1: 6$ in the visual cortex (Shadlen \& Newsome, 1994; Abeles, 1990), the requirement $\lambda_{G_{A}}>\lambda_{A}$ implies that each $\mathrm{GABA}_{A}$ synapse fires much faster than each AMPA synapse.

In all the following numerical simulations, we assume that the neurotransmitter at the presynaptic terminal is released as a Poisson process with a rate $\lambda$ [see eqn (6) and (14)].

With the given parameters in Appendix B, a calculation using the previous results tells that when

$$
\lambda_{G_{A}}=276 \times \lambda_{A} \mathrm{~Hz}
$$

a post-synaptic exact balance input is attained provided that $\bar{g}_{A}=\bar{g}_{G_{A}}$. As we have pointed out before, a much faster input at each $\mathrm{GABA}_{A}$ synapse than at each AMPA synapse is required for balance (see Fig. 3). Interestingly, we note that when AMPA and $\mathrm{GABA}_{A}$ reach an exact balance in the stationary region, an almost exact balance in the transitional phase is also achieved since $\beta_{A} \sim \beta_{G_{A}}$ (see Lemma A.1). This is quite a remarkable result.

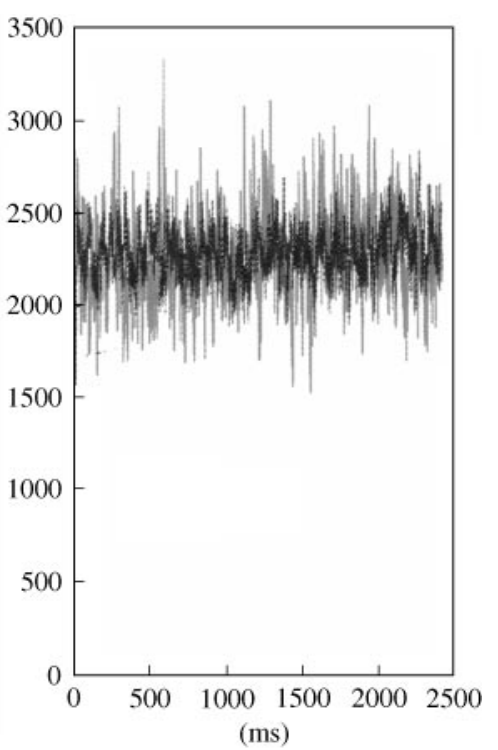

FIG. 3. $\bar{I}_{A}(t) / \bar{g}_{A}=r_{A}(t)\left(E_{A}-V_{\text {rest }}\right)(\mathrm{AMPA})$ and $-\bar{I}_{G_{A}}(t) / \bar{g}_{G_{A}}=r_{G_{A}}(t)\left(V_{\text {rest }}-V_{G_{A}}\right)\left(\mathrm{GABA}_{A}\right)$ vs. time. (Left) $\lambda_{A}=10000 \mathrm{~Hz}$ (see, for example, Feng \& Brown, 2000) for the choice of this parameter) and $\lambda_{G_{A}}=7000 \mathrm{~Hz}$, horizontal lines are estimated mean, i.e. $650(1-\exp (-1.1)) / 0.19$ and $105(1-\exp (-5.0)) / 0.18$. (Right) $\lambda_{A}=10000 \mathrm{~Hz}$ and $\lambda_{G_{A}}=27600 \mathrm{~Hz}$, and an exact balance between inputs at the post-synaptic site is reached. 
Now we turn to consider how the behaviour of the integrate-and-fire is affected by the balanced input with parameters $\gamma=20.2 \mathrm{~ms}, V_{\text {rest }}=$ $-65 \mathrm{mV}, V_{\text {thre }}=-50 \mathrm{mV}$. We also simulate the model with Poisson point process inputs with reversal potentials, i.e. $\bar{r}(t)=\delta(t)$ the delta function at time $t$. In Fig. 3, the IF model is simulated with a fixed excitatory input rate of $10000 \mathrm{~Hz}$ and varying inhibitory input rates. A few interesting phenomena we observe here are as follows.

- As we might expect, the mean interspike intervals with AMPA and $\mathrm{GABA}_{A}$ synaptic input is lower than with Poisson point process inputs. This could be understood from the following calculations. Suppose that a dynamical system $y_{t}$ with a decay $L$ is described by $\mathrm{d} y_{t}=$ $-L y_{t} \mathrm{~d} t+h(t) \mathrm{d} t$ with $\int h(t) \mathrm{d} t=1$ and $h \geqslant 0$, which is roughly the case of our consideration. Then we have $y_{t}=\int_{0}^{t} \exp (-L(t-s)) h(s) \mathrm{ds}$ $\leqslant 1$. The equality holds if and only if $h(s)=\delta_{0}(s)$. When $h(s)=\delta_{0}(s)$ is the Poisson (instantaneous) input case. In words, suppose the system receives an input of total amount of 1. Because of the decay mechanism, the strongest input for the system to receive is instantaneous input (without taking any time).

- Nevertheless, at high firing rates the integrateand-fire model with Poisson point process inputs is a very good approximation to the model with AMPA and $\mathrm{GABA}_{A}$ inputs. For lower output rates, the approximation becomes worse. It indicates that for more balanced inputs we have to resort to the original, more complex mechanism to simulate the model behaviour.

- There is a region in which the output $\mathrm{CV}$ is greater than 0.5. Many studies have been devoted to the problem of how to generate spike trains with $\mathrm{CV}$ between 0.5 and 1 , which is observed from the experimental data (Softky \& Koch, 1993). In particular, in Softky \& Koch (1993), the authors point out that it is impossible for the IF model to generate spike trains with CV between 0.5 and 1 . Many approaches have been proposed to get around this problem (Shadlen \& Newsome, 1994). Here, as we have demonstrated elsewhere (Feng \& Brown, 2000), it is shown that the integrate-and-fire model is capable of emitting spike trains with $\mathrm{CV}$ greater than 0.5 and it is not necessary to go to the case of exactly balanced inputs (Shadlen \& Newsome, 1994).

- An exact post-synaptic balance is never reached in the physiological parameter range, since the output mean interspike interval becomes too long, even before an exact pre-synaptic balance occurs. For example, when the inhibitory input is around $8000 \mathrm{~Hz}$, the output firing rate is $2 \mathrm{~Hz}$ (see Fig. 3), without even adding a refractory period. We then see that 5000 excitatory synapses are needed provided that input rates equal output rates (see Abbott et al., 1997). This number is even higher than the total number of excitatory synapses connected to a cell, estimated to be around 3000 (Shadlen \& Newsome, 1994).

- The integrate-and-fire model exhibits the "push-pull" effect: with a large mean interspike interval, the output $\mathrm{CV}$ is also high [see Fig. 4 (right)].

\subsection{NMDA AND GABA ${ }_{B}$ SYNAPSES}

Based upon an approach like that above, we need to estimate when

$$
\left\langle I_{s y n}(t)\right\rangle=\left\langle I_{N}(t)\right\rangle+\left\langle I_{G_{B}}(t)\right\rangle=0 .
$$

However, because of the effects of magnesium block represented by $B(V)$ we are not able to get an exact estimate as in the previous subsection. We approximately consider

$\bar{g}_{N}\left\langle r_{N}\right\rangle B\left(V_{\text {rest }}\right)\left(E_{N}-V_{\text {rest }}\right)=\bar{g}_{G_{B}}\left\langle\frac{s^{n}}{s^{n}+K}\right\rangle\left(V_{\text {rest }}-E_{G_{B}}\right)$

which again means a local balance: if the membrane potential is at the resting level then NMDA inputs are balanced with $\mathrm{GABA}_{B}$ inputs.

As shown in Fig. 5, we see that $s^{4}(t) /$ $\left(s^{4}(t)+K_{1}\right)$ rapidly converges to 1 , irrespective of whether the input is 100 or $1000 \mathrm{~Hz}$. Fig. 6 shows $\bar{I}_{N}=\bar{g}_{N} r_{N}(t) B\left(V_{\text {rest }}\right)\left(E_{N}-V_{\text {rest }}\right)$ and $-\bar{I}_{G_{B}}=$ $\bar{g}_{G_{B}} r_{G_{B}}(t)\left[s^{4}(t) /\left(s^{4}(t)+K_{1}\right)\right]\left(V_{\text {rest }}-E_{G_{B}}\right)$. Therefore, for $\mathrm{GABA}_{B}$ inputs, different from the other three inputs which sum linearly (summation of Poisson processes is again a Poisson process), we have to use multi-synapse simulation to simulate multi-synaptic $\mathrm{GABA}_{B}$ inputs. 

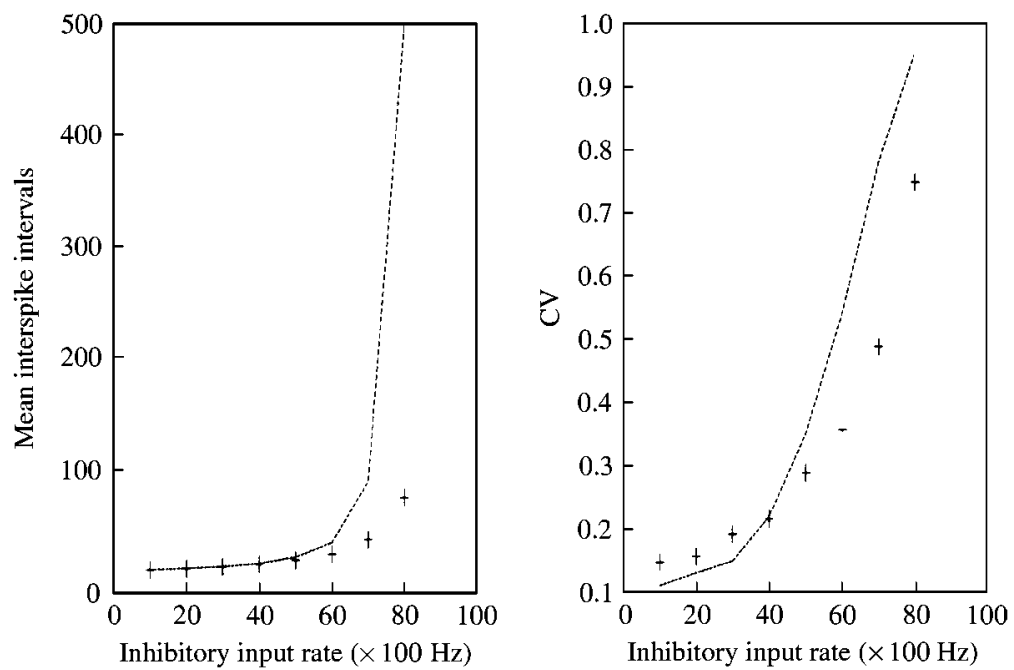

FIG. 4. Mean interspike interval vs. inhibitory synaptic $\left(\mathrm{GABA}_{A}, \ldots-\ldots\right)$ input rate. Data are obtained for averaging over 2000 interspike intervals. $\bar{g}_{A}=\bar{g}_{G_{A}}=0.001$. Approximation $(+)$ indicates data obtained for the model with Poisson inputs. The mean interspike interval is too large even before an exact pre-synaptic balance is reached.
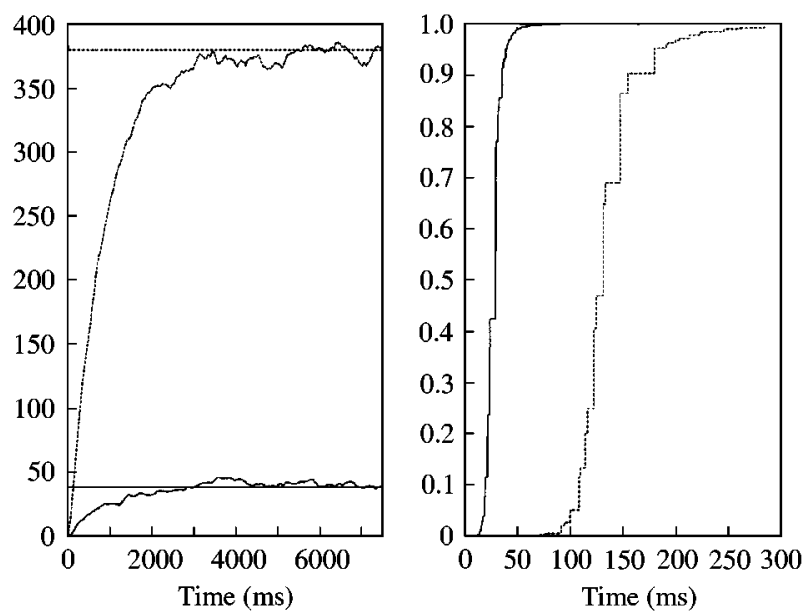

FIG. 5. $s(t)$ (left) and $s^{4}(t) /\left(s^{4}(t)+K_{1}\right)$ (right) vs. time with $\lambda=100(--)$ and $1000(-)$ Hz. In both cases $s^{4}(t) /\left(s^{4}(t)+K_{1}\right)$ reaches its saturated value rapidly, although $s(t)$ is a stochastic process.

\subsection{GENERAL CASES}

In general, an exact post-synaptic balance requires that

$$
\left\langle I_{A}\right\rangle+\left\langle I_{N}\right\rangle+\left\langle I_{G_{A}}\right\rangle+\left\langle I_{G_{B}}\right\rangle=0 .
$$

If we assume $\lambda_{A}=\lambda_{N}$, the remaining free parameters are $\lambda_{G_{A}}$ and $\lambda_{G_{B}}$ which we would then require to satisfy

$\bar{g}_{G_{A}}\left\langle r_{G_{A}}\right\rangle\left(V_{\text {rest }}-E_{G_{A}}\right)+\bar{g}_{G_{B}}\left\langle\frac{s^{n}}{s^{n}+K_{1}}\right\rangle\left(V_{\text {rest }}-E_{G_{B}}\right)$

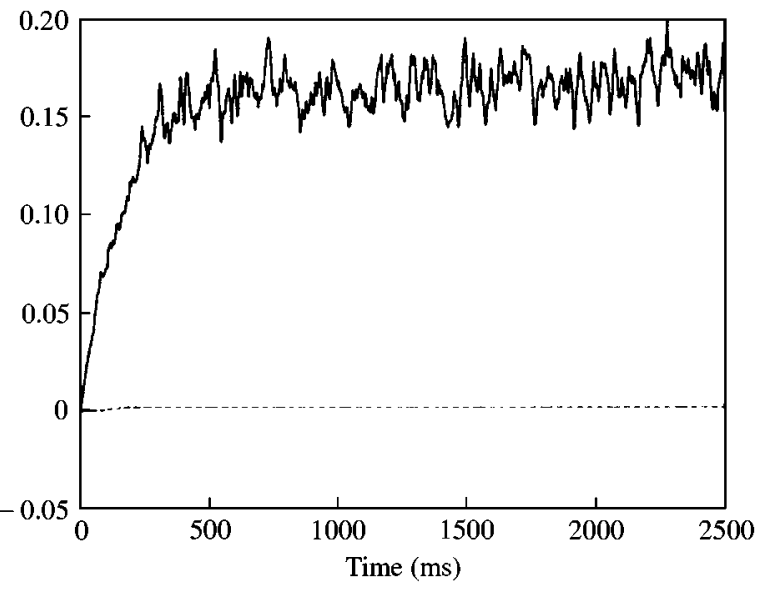

FIG. 6. $\bar{I}_{N}$ and $-\bar{I}_{G_{B}}$ vs. time with $\lambda_{N}=10000 \mathrm{~Hz}$ and $\lambda_{G_{B}}=100 \mathrm{~Hz}$. As we have shown in Fig. 5, increasing $\lambda_{G_{B}}$ has almost no effect on $-\bar{I}_{G_{B}}$ : $(-)$ NMDA; (-----) GABA G. .

$$
=\bar{g}_{A}\left\langle r_{A}\right\rangle\left(E_{A}-V_{\text {rest }}\right)+\bar{g}_{N} B\left(V_{\text {rest }}\right)\left\langle r_{A}\right\rangle\left(E_{N}-V_{\text {rest }}\right) .
$$

Now, we carry out numerical simulations of the integrate-and-fire model with all four types of synaptic input. For AMPA, NMDA and GABA $A$ inputs, we can simply use one synaptic input with higher firing rate to replace multi-synaptic inputs. However, for $\mathrm{GABA}_{B}$ we assume that $N_{G_{B}}$ synapse are presented.

Fig. 7 shows that for the model with four types of synaptic input, we can approximately treat 


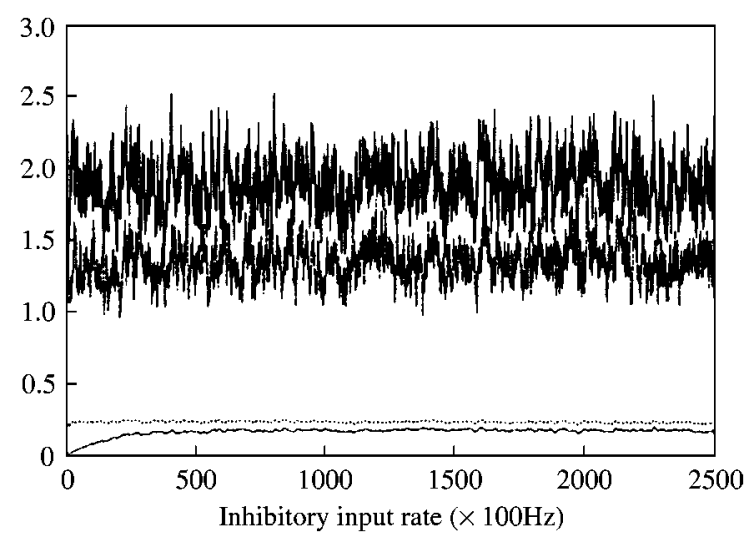

FIG. 7. $\bar{I}_{A},-\bar{I}_{G_{A}},-\bar{I}_{G_{B}}$ and $\bar{I}_{N}$ (from top to bottom) vs. time with $\lambda_{A}=\lambda_{N}=\lambda_{G_{A}}=9000 \mathrm{~Hz}$ and $\lambda_{G_{B}}=100 \mathrm{~Hz}$, $N_{G_{B}}=90$ : ( $(--)$ NMDA.

NMDA and $\mathrm{GABA}_{B}$ inputs as DC currents since, compared with AMPA and $\mathrm{GABA}_{A}$ inputs, their fluctuations are much smaller.

We simulated the model with four and three types of synaptic inputs, $\mathrm{GABA}_{B}$ being excluded in the latter case. As we could expect of the model with positive DC inputs, CV is low compared with the model with negative DC inputs. The reason is simply that the position of the attractor of the deterministic part of the model is higher with positive DC inputs than that with negative DC inputs. Therefore, with large
$\mathrm{GABA}_{B}$ inputs, a large $\mathrm{CV}$ of the efferent spike trains is obtained.

However, we note from Fig. 8 that when output $\mathrm{CV}$ is greater than 0.5 , the mean interspike interval is about $50 \mathrm{~ms}$, much greater than input intervals. In general, we would expect that noninstantaneous inputs such as the case we studied in this paper are weaker than Poisson inputs. Whether it is possible to find a set of physiologically plausible parameters such that the input and output rates are similar, but the output $\mathrm{CV}$ is greater than 0.5 . We go to the extreme case by assuming that 1000 synapses fire at a rate of 100 Hz. Fig. 9 depicts numerical results. All conclusions above remain.

\section{Discussion}

In this paper, we consider the integrateand-fire model with four type of synaptic input. Analytical expressions for the post-synaptic current are derived. AMPA, NMDA and $\mathrm{GABA}_{A}$ synapses are the simplest cases, involving a single-gating variable, $r$, although NMDA has the additional complication of magnesium block, which incorporates an additional membrane potential dependence. $\mathrm{GABA}_{B}$ synapses are more complex involving a nonlinear dependence on the gating variable. This results in the additional
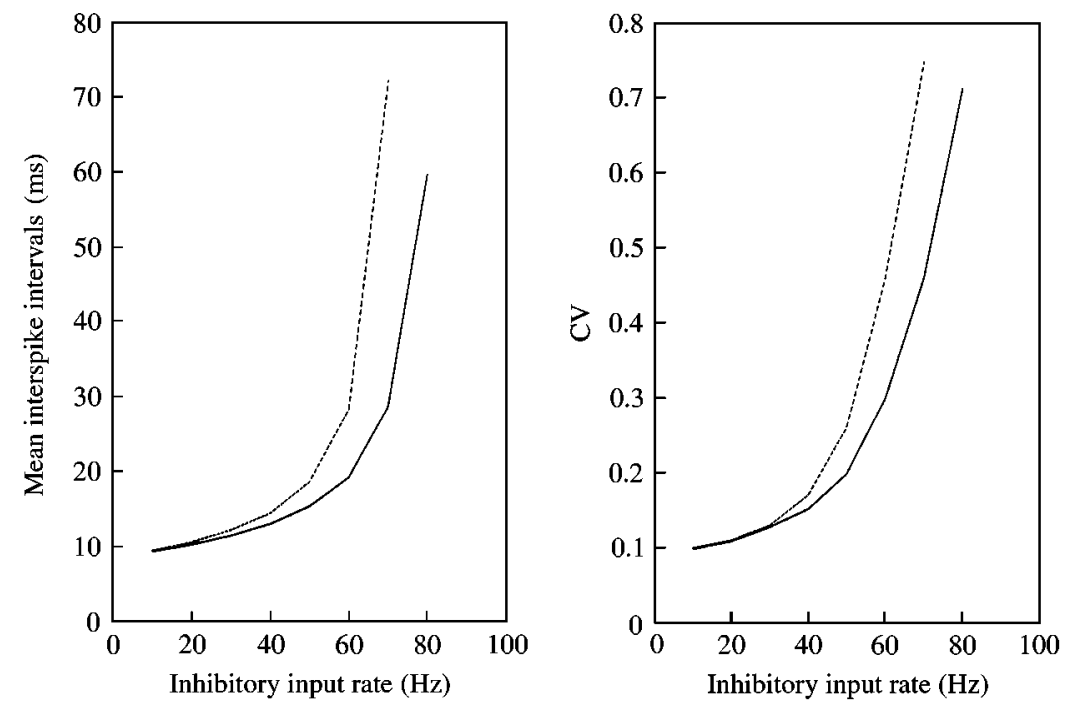

FIG. 8. Four (-): Mean interval and $\mathrm{CV}$ of efferent spike trains with four types of synaptic input with $\lambda_{A}=\lambda_{N}=10000 \mathrm{~Hz}$ (total frequencies over all synapses) vs. $\lambda_{G_{A}}=\lambda_{G_{B}}=0,10,20, \ldots, 100 \mathrm{~Hz}$ and $N_{G_{B}}=N_{G_{A}}=100$. Three $(-----)$; parameters are as in Four except that $N_{G_{B}}=0$. 

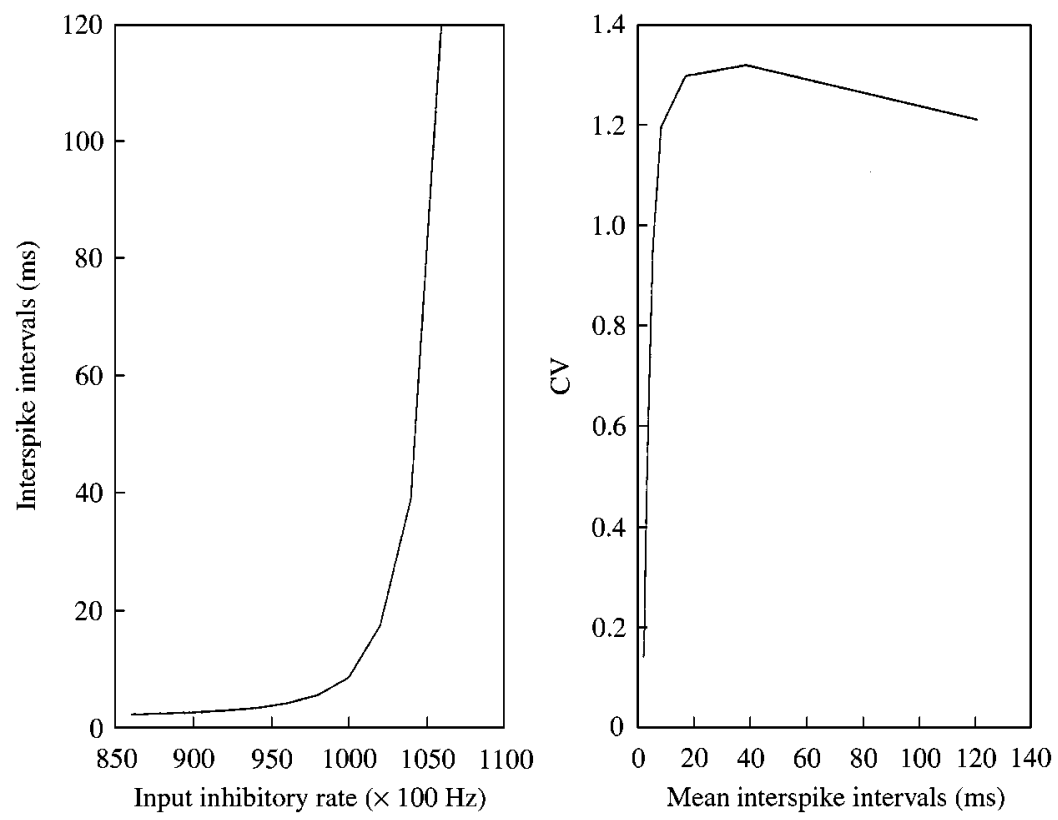

FIG. 9. Mean (-) firing rate vs. inhibitory input rate with $\lambda_{G_{A}}=\lambda_{G_{B}}=850,900, \ldots, 1100 \mathrm{~Hz}, N_{G_{A}}=N_{G_{B}}=100$, $\lambda_{A}=\lambda_{N}=1000 \mathrm{~Hz}$ and $N_{A}=N_{N}=100$ (left). CV (-) of efferent spike trains vs. mean interspike intervals (right).

complication compared to the other three that it easily saturates, and so synaptic inputs cannot be pooled for simplicity as they can for the other synaptic types. NMDA and $\mathrm{GABA}_{B}$ inputs are in a sense simpler than $\mathrm{GABA}_{A}$ and AMPA as they can be treated as DC currents, since the fluctuations in current resulting from them are small in comparison to $\mathrm{GABA}_{A}$ and AMPA.

The possibility of exactly balanced inputs is examined for the integrate-and-fire model. Exact post-synaptic balance could only ever be an approximation, since whether the excitatory and inhibitory inputs balance depends on membrane potential. At some membrane potentials they balance, and at others they do not. However, for $\mathrm{GABA}_{A}$ and AMPA synapses, the input levels which result in exact balance at the resting potential can be determined, and this might form a usable approximation, since neuronal membrane potential is often close to resting potential for much of the time.

However, probably a better approach is to think of post-synaptic balance as a statistical phenomenon, i.e. that excitatory and inhibitory inputs balance over a period of time for particular input stochastic processes. Conditions are derived here for the balance during the stationary phase, in the simple case of AMPA and $\mathrm{GABA}_{A}$ synapses using Lemma 1. This suggests that, in view of substantial differences in the effects of reversal potentials and of the inequality between the numbers of inhibitory and excitatory synapses (about 1:6 (Shadlen \& Newsome, 1994; Abeles, 1990) in the visual cortex, for example, each $\mathrm{GABA}_{A}$ synapse would need to fire much faster than the AMPA synapses to achieve balance. Conditions could similarly be derived for balance between NMDA and $\mathrm{GABA}_{B}$ since they are approximately equivalent to constant currents.

Balanced inputs between $\mathrm{GABA}_{A}$ and AMPA do not occur either pre-synaptically or postsynaptically for the leaky integrator model parameters we used in this paper, because the output mean interspike interval becomes too long before this point is reached. The functional implications of a net positive input are clear, in comparison with a zero input (exactly balanced inputs): it keeps the neuron at a level more easily to fire, or more quickly to react to input signals. Our approach is based upon theoretical results and so is general. With the availability of newly, more explicit experiment data, we could easily perform similar calculations as we presented here. Finally, 
the discussion on balanced inputs at pre- or postsynaptic sites is independent of neuronal models.

The work was financially supported by the BBSRC, by EU grant number Bio4-98-0135 and the Royal Society.

\section{REFERENCES}

Aвbott, L. F., Varela, J. A., Sen, K. \& Nelson, S. B. (1997). Synaptic depression and cortical gain control. Science 275, 220-223.

Abeles, M. (1990). Corticonics. Cambridge: Cambridge University Press.

Brown, D. \& FENG, J. (1999). Is there a problem matching model and real CV (ISI)? Neurocomputing 26-27, 117-112.

Brown, D., FEnG, J. \& FEERICK, S. (1999). Variability of firing of Hodgkin-Huxley and FitzHugh-nagumo neurones with stochastic synaptic input. Phys. Rev. Lett. 82, 4731-4734.

De Koninck, Y. \& Mody, I. (1994). Noise analysis of miniature IPSCs in adult rat brain slices: properties and modulation of synaptic $\mathrm{GABA}_{A}$ receptor channels. $J$. Neurophysiol. 71, 1318-1335.

Destexhe, A., Mainen, Z. F. \& Sejnowski, T. J. (1998). Kinetic models of synaptic transmission. In: Methods in Neuronal Modelling (Koch, C. \& Segev, I., eds), pp. 1-26. Cambridge, MA: MIT Press.

FENG, J. (1997). Behaviours of spike output jitter in the integrate-and-fire model. Phys. Rev. Lett. 79, 4505-4508.

FENG, J. (1999). Origin of firing variability of the integrateand- fire model. Neurocomputing 26-27, 87-91.

FENG, J. \& BROWN, D. (1998a). Spike output jitter, mean firing time and coefficient of variation. J. Phys. A: Math. Gen. 31, 1239-1252.

FENG, J. \& BROWN, D. (1998b). Impact of temporal variation and the balance between excitation and inhibition on the output of the perfect integrate-and-fire model. Biol. Cybern. 78, 369-376.

FENG, J. \& BROWN, D. (1999). Coefficient of variation greater than 0.5. How and when? Biol. Cybern. 80, 291-297.

FENG, J. \& BROWN, D. (2000). Impact of correlated inputs on the output of the integrate-and-fire models. Neural Comput. 12, 711-732.

FenG, J., Brown, D. \& LI, G. (2000). Synchronization due to common pulsed input in Stein's model. Phys. Rev. E 61, 2987-2995.

Harris, C. M. \& Wolpert, D. M. (1998). Signal-dependent noise determines motor planning. Nature 394, 780-784.

JAHR, C. E. \& STEVENS, C. F. (1990). A quantitative description of NMDA receptor-channel kinetic behaviour. $J$. Neurosci. 10, 1830-1837.

KANDEl, E. R., Schwartz, J. H. \& Jessell, T. M. (1991). Principles Of Neural Science, 3ed Edn, Englewood Cliffs, $\mathrm{NJ}$ : Prentice-Hall International Inc.

Konig, P., ENGEL, A. K. \& Singer, W. (1996). Integrator or coincidence detector? The role of the cortical neuron revisited. Trans. Inst. Neurosci. 19, 130-137.

MUSILA, M. \& LÁNSKÝ, P. (1994). On the interspike intervals calculated from diffusion approximations for Stein's neuronal model with reversal potentials. J. theor. Biol. 171, $225-232$.
Otis, T. S., De Koninck, Y. \& Mody, I. (1992). Whole-cell recordings of evoked and spontaneous $\mathrm{GABA}_{B}$ responses in hippocampal slices. Pharmacol. Commun. 2, 75-83.

RiCCIARDI, L. M. \& SATO, S. (1990). Diffusion process and first-passage-times problems. In: Lectures in Applied Mathematics and Informatics (Ricciardi, L. M., ed.). Manchester: Manchester University Press.

Shadlen, M. N. \& Newsome, W. T. (1994). Noise, neural codes and cortical organization. Curr. Opin. Neurobiol. 4, $569-579$.

SofTKY, W. \& KoCH, C. (1993). The highly irregular firing of cortical-cells is inconsistent with temporal integration of random EPSPs. J. Neurosci. 13, 334-350.

Thomson, A. M. (1997). Activity-dependent properties of synaptic transmission at two classes of connections made by rat neocortical pyramidal. J. Physiol. 502, 131-147.

Troyer, T. W. \& Miller, K. D. (1997). Physiological gain leads to high ISI variability is a simple model of a cortical regular spiking cell. Neural Comput. 9, 733-745.

TuCKWEll, H. C. (1988). Stochastic Processes in the Neurosciences. Philadelphia, PA: Society for Industrial and Applied Mathematics.

Van Vereeswijk, C., Abbott, L. F. \& Ermentrout, G. B. (1994). J. Comput. Neurosci. 1, 313-321.

Wilbur, W. J. \& Rinzel, J. (1983). A theoretical basis for large coefficient of variation and bimodality in neuronal interspike interval distributions. $J$. theor. Biol. 105, 345-368.

Xiang, Z., Greenwood, A. C. \& Brown, T. (1992). Measurement and analysis of hippocampal mossy-fiber synapses. Soc. Neurosci. Abstr. 18, 1350.

Zigmond, M. J., Bloom, F. E., Landis, S. C., Roberts, J. L. \& SQUIRE, L. R. (1999). Fundamental Neuroscience. San Diego, London: Academic Press.

\section{APPENDIX A}

The following lemma is proved in Feng et al. (2000).

Lemma A.1. For $\tilde{r}(t)$ defined as before we have

$$
\tilde{r}(t) \mathrm{d} t \sim \mu(t)\left(\mu \mathrm{d} t+\sigma \mathrm{d} B_{t}+c \mathrm{~d} \xi_{t}\right)
$$

where $B_{t}$ is the standard Brownian motion, $\xi_{t}$ is an Ornstein-Uhlenbeck $(O U)$ process, $c$ is a constant

$$
\mu(t)=1-\int_{t}^{\infty} \tilde{r}(u) \mathrm{d} u
$$

and $\mu=\bar{r} \lambda, \sigma^{2}=\bar{r}^{2} \lambda$, where $\lambda$ is the total rate of incoming EPSCs or IPSCs.

\section{APPENDIX B}

\section{Parameter Values}

The following parameter sets are taken from Destexhe et al. (1998). For AMPA synapses (recorded from Hippocampal mossy-fiber synapses 
(Xiang et al., 1992), we use $\alpha_{A}=1.1 \mathrm{~ms}^{-1} \mathrm{mM}^{-1}, \quad \alpha_{N}=0.072 \mathrm{~ms}^{-1} \mathrm{mM}^{-1}, \quad \beta_{N}=0.0066 \mathrm{~ms}^{-1}$, $\beta_{A}=0.19 \mathrm{~ms}^{-1}, E_{A}=0 \mathrm{mV}$ which gives $\bar{r}_{A}=E_{N}=0 \mathrm{mV}$; for $\mathrm{GABA}_{B}$ synapses (recorded from 3.51; for $\mathrm{GABA}_{A}$ synapses (recorded from Hip- rat Hippocampal slices (Otis et al. 1992)), pocampal slices of rat (De Koninck \& Mody, $\alpha_{G_{B}}=0.09 \mathrm{~ms}^{-1} \mathrm{mM}^{-1}, \beta_{G_{B}}=0.0012 \mathrm{~ms}^{-1}, K_{1}=$ 1994) $\alpha_{G_{A}}=5 \mathrm{~ms}^{-1} \mathrm{mM}^{-1}, \quad \beta_{G_{A}}=0.18 \mathrm{~ms}^{-1}, \quad 100, \quad K_{2}=0.18, \quad K_{3}=0.034, \quad E_{G_{B}}=-95 \mathrm{mV}$, $E_{G_{A}}=-80 \mathrm{mV}$ which gives $\bar{r}_{G_{A}}=5.52 ;$ for and $\bar{g}_{A}=0.001, \quad \bar{g}_{G_{A}}=0.001, \quad \bar{g}_{N}=0.0003$, NMDA synapses (see Jahr \& Stevens, 1990), $\quad \bar{g}_{G_{B}}=0.00006$. 